\title{
Concurrent crystallization of multiple proteins in a single cell without interfering each other's phase separation events
}

\author{
Haruki Hasegawa \\ Amgen Inc, South San Francisco, United States of America \\ harukih@amgen.com
}

Intracellular protein crystallization occurs in many branches of life, yet the underlying cellular processes remain largely unknown. This is partly because of the scarcity of easily accessible, reproducible recombinant protein models that allow in-depth characterization of intracellular liquid-solid phase separation events. Such limitation prompts the need for identifying various classes of model proteins to examine the similarities, differences, or generalizability of such intracellular crystallization events. Furthermore, to exploit the potential values of cell-made protein crystals and the platforms to produce them, intracellular crystallization should first be understood using diverse classes of model proteins. After validating the individual crystallization events of cellular and viral proteins that readily crystallize in the ER, cytosol or nucleus, I demonstrate up to four independent crystallization events can take place concurrently in various combinations in different subcellular compartments of a single cell. For instance, by co-expressing NEU1 and human IgGs that undergo crystallization or liquid-liquid phase separation in the ER, I demonstrate two independent phase separation events can be simultaneously induced in the same continuous space of the ER lumen without mixing or interfering each other's phase separation behaviors. Likewise, two concurrent crystallization events can take place in the cytosol or in the nucleus without mixing or interfering each other. Intracellular protein crystallization thus can happen in a crowded physiological cellular environment and does not require high protein purity. Furthermore, I report a simple method to increase the yield of intracellular protein crystals, in terms of crystal size and numbers, by treating the cells with a topoisomerase II inhibitor that blocks cell division without preventing cell size growth. This study not only presents accessible model tools for studying intriguing intracellular protein crystallization events, but also paves a way toward establishing methods and controlling the induction, quality, size, and yield of intracellular protein crystals for high-value proteins.

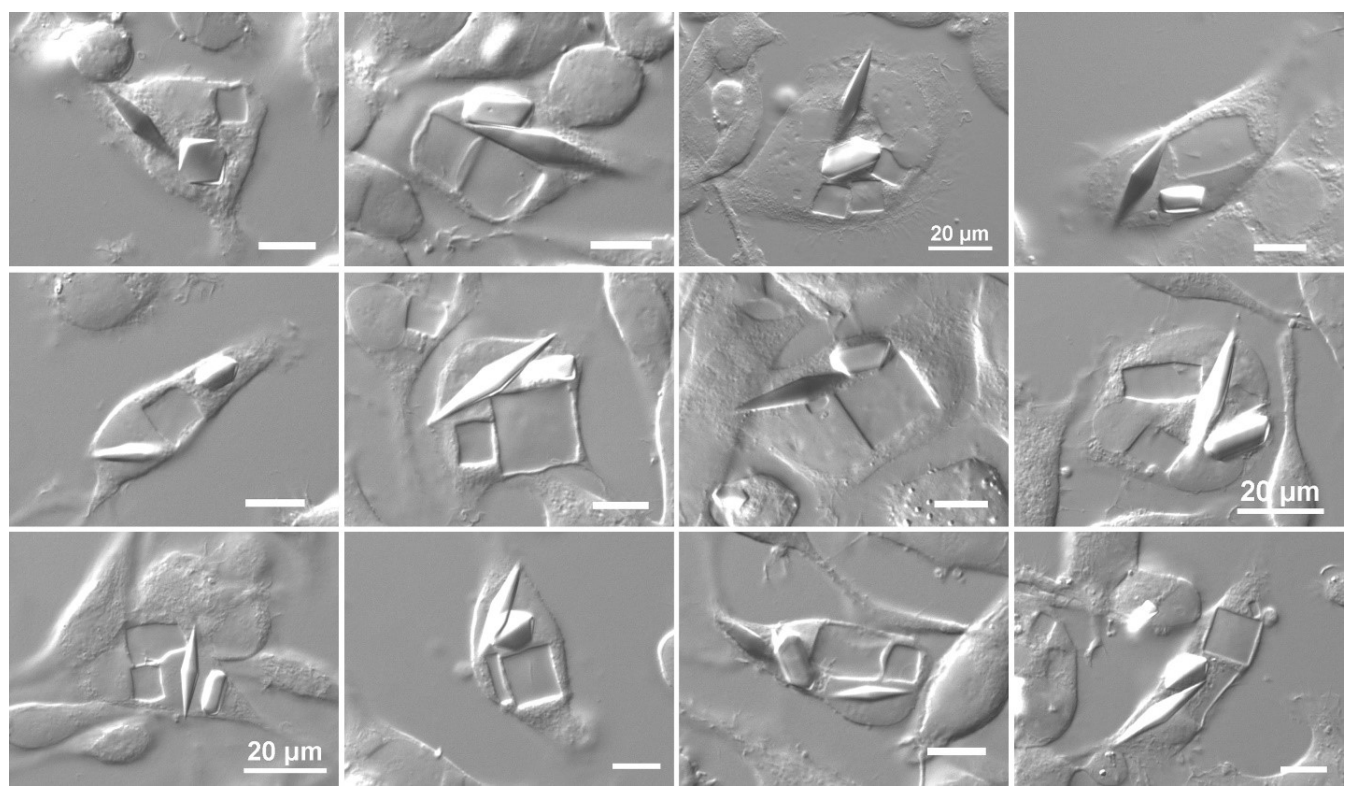

Figure 1. Induction of three independent phase separation events in different subcellular compartments of a single cell. DIC micrographs of HEK293 cells co-expressing three model proteins NEU1, CLC protein, and CRYGD-R37S variant, simultaneously. Images were captured on day-5 post transfection. Unlabeled scale bar represents $10 \mu \mathrm{m}$.

Keywords: intracellular protein crystallization, phase separation, ER, cytosol, nucleus 